\title{
TP53 Positive
}

National Cancer Institute

\section{Source}

National Cancer Institute. TP53 Positive. NCI Thesaurus. Code C146668.

An indication that TP53 expression has been detected in a sample. 